\title{
Selective Sensing of Cannabis Odor Using a Thermal Shock-Induced Tin Oxide Gas Sensor
}

\author{
Faramarz Hossein-Babaei, Maryam Ebrahimi \\ Electronic Materials Laboratory, Electrical Engineering Department, K. N. Toosi University of \\ Technology, Tehran, 16317-14191, Iran \\ fhbabaei@kntu.ac.ir
}

\begin{abstract}
:
Detection and concentration estimation of tetrahydrocannabinol (THC), the active ingredient in cannabis, is an urgent technical challenge because of road safety issues and health/legal concerns. It is particularly important to differentiate this substance from other volatile organic compounds (VOCs) in air and breath. Here, we report using a virtual sensor array made by a thermal shock-induced tin oxide gas sensor for the detection, recognition, and concentration estimation of THC in air. The presented sensory system is capable of distinguishing THC from VOCs such as ethanol, propanol, acetone, and toluene, and can be trained to perform analyses on binary alcoholTHC gas mixtures.
\end{abstract}

Key words: Cannabis, odor detection, artificial olfaction, quasi-sensor array, Selective VOC detection

\section{Introduction}

The severe health, economical, moral, and legal impacts of cannabis on the society necessitates easily accessible and effective detection devices [1]. Despite their accuracy, classic analytical systems are expensive and heavy and usually require expert operators.

Sensor array-based electronic nose systems are compact and user friendly, and may seem suitable for the task, however, unpredictable drifts of their array components may render them unreliable. Alternative techniques have been developed for creating a virtual array from a single general sensor. Operating temperature modulation of the sensor is the most widely investigated technique [2]. A more recent version of the temperature modulated sensor, thermal shock-induced (TSI) gas sensor, has been demonstrated to provide more uncorrelated data on the analyte $[3,4]$.

Here, we report the detection, recognition and concentration determination of THC in air utilizing a virtual sensor array made of a TSI tin oxide gas sensor. The system is capable of determining THC at the presence of other volatile organic vapors such as ethanol. Our prototype cannabis detector is easy to use, battery-operated, miniaturized, and linked to a laptop for fast data processing.

\section{Experimental}

A virtual sensor array is formed by operating a single tin oxide chemoresistive gas sensor at different operating temperatures provided by a ruthenium dioxide microheater. The structure of the sensor is schematically presented in Fig. 1a.
By applying a nominal working voltage of $5 \mathrm{~V}$ to the heater, its surface temperature is brought to $300{ }^{\circ} \mathrm{C}$. The sensor head is then introduced into the test chamber containing the analyte contaminated air. The voltage waveform shown in Fig. $1 \mathrm{~b}$ is, applied to the microheater, which starts with a step fall from $5 \mathrm{~V}$ to $1 \mathrm{~V}$ level. This is followed by the voltage impulses each inducing a thermal shock to the sensing pellet of the sensor. The heating waveform is $3 \mathrm{~s}$ long, and upon its completion, the heating voltage, as shown in Fig. $1 \mathrm{~b}$ returns back to the original operating point at $5 \mathrm{~V}$, and the sensor head is brought out from the chamber to clean air for recovery at its nominal operating temperature.

The information regarding the pyrolysis of the analyte molecules becomes available through the recorded transient responses of the sensor. The schematic diagram of the measurement system is depicted in Fig. 1c.

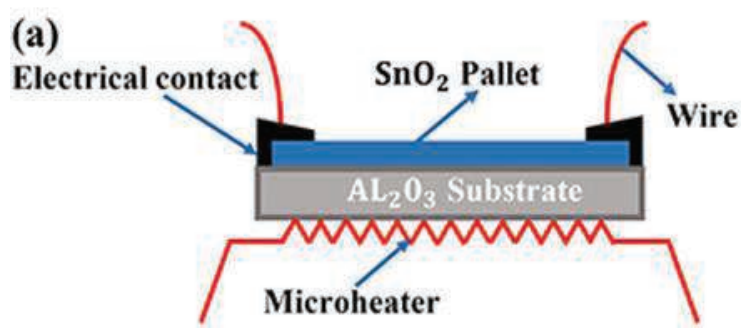


(b)

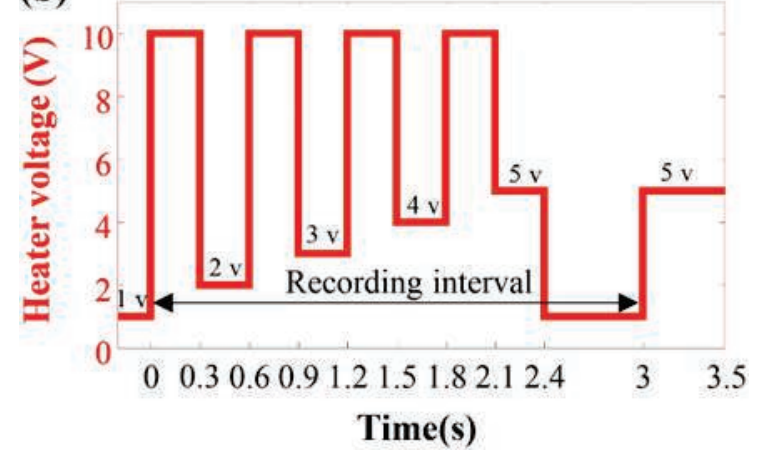

(c)

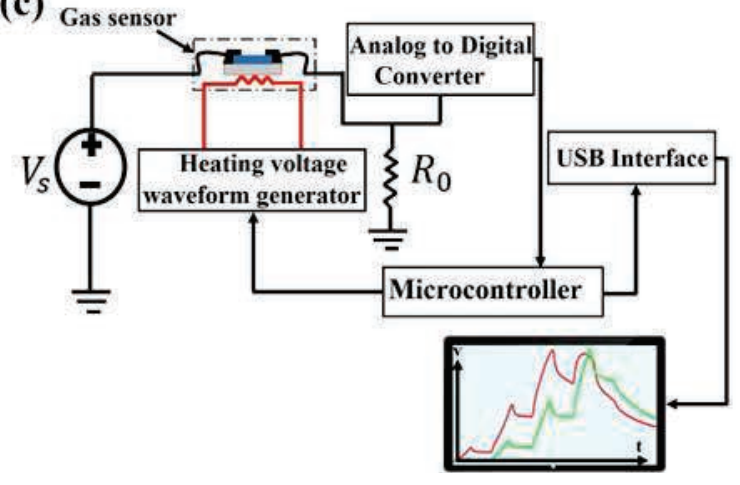

Fig. 1. (a) Schematic diagram of the sensor; (b) the waveform applied to the microheater of the sensor; (c) schematic diagram of the measurement system.

The response of the temperature-modulated sensor to an analyte contaminated air is defined as the temporal variations of the sensor resistance $\left(R_{s}(t)\right)$ during the time frame of the heating waveform. Prior to each test, the base line of the array, defined as its response to clean air, is recorded and deducted from all recorded temporal responses.

A 300 dimensional feature vector is heuristically formed for each analyte. Feature vector components are taken from the peaks, plateaus, slopes, and deflection points present on each response pattern, which define the profile of the response. The principal component analysis (PCA) technique is used for dimension reduction and visualization in a three-dimensional feature space. The results are presented in Fig. 2.

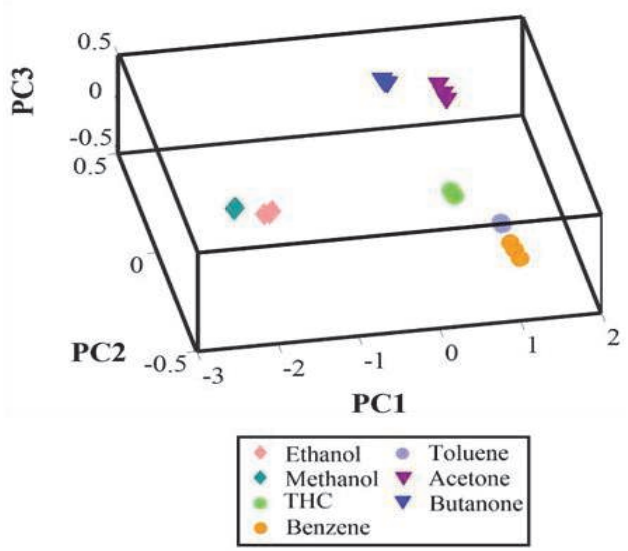

Fig. 2. Feature space presentation resulted from PCA mapping of the feature vectors related to the recorded response patterns demonstrating the segregation of the different classes.
To evaluate the degree of interference of ethanol in THC detection and identification, response patterns are recorded for the different mixtures of the (THC) $)^{-}$ (ethanol) 1-x type, wherein $x$ varies from zero to one. The recorded responses for different $x$ values were mapped into the feature space using the above described method. Results are given in Fig. 3.

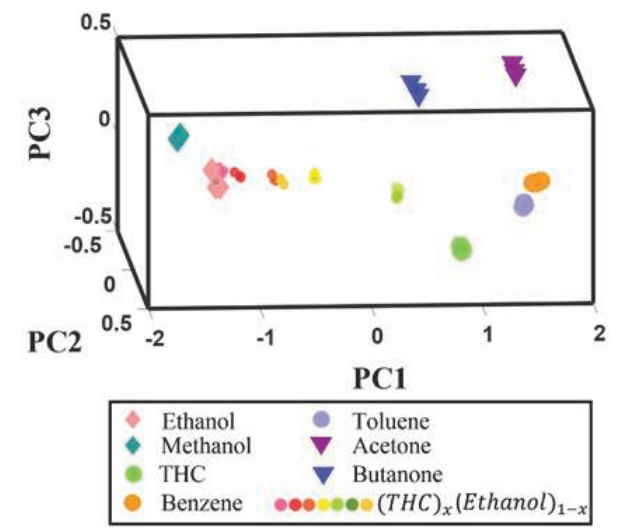

Fig. 3. Feature space presentation showing the classification of $(T H C)_{x}(\text { Ethanol })_{1-x}$ responses.

\section{Conclusion}

We successfully used a thermal shock-induced resistive tin oxide gas sensor for the selective detection and concentration evaluation of the cannabis odor in air.

\section{References}

[1] Hall, W., and M. Weier. "Assessing the public health impacts of legalizing recreational cannabis use in the USA." Clinical pharmacology \& therapeutics 97.6 (2015): 607-615. DOI: $10.1002 /$ cpt. 110

[2] Hossein-Babaei, F., S. Hosseini-Golgoo, and A. Amini, Extracting discriminative information from the Padé-Z-transformed responses of a temperature-modulated chemoresistive sensor for gas recognition. Sensors and Actuators B: Chemical, 2009. 142(1): p. 19-27. DOI: doi.org/10.1016/j.snb.2009.07.039

[3] Hossein-Babaei, F. and A. Amini, A breakthrough in gas diagnosis with a temperature-modulated generic metal oxide gas sensor. Sensors and Actuators B: Chemical, 2012. 166: p. 419-425. DOI: doi.org/10.1016/j.snb.2012.02.082

[4] Hossein-Babaei, F. and A. Amini, Recognition of complex odors with a single generic tin oxide gas sensor. Sensors and Actuators B: Chemical, 2014. 194: p. 156-163. DOI: doi.org/10.1016/j.snb.2013.12.061 